\title{
Towards A Multi-dimensional Biometric Approach to Quantifying Student Engagement in the STEM Classroom
}

\section{Dr. James Christopher Foreman, University of Louisville}

Asst. Professor at University of Louisville, previous appointment at Purdue University. Teaching calculus, power and energy, and industrial control systems related courses. Research in artificial neural networks, expert systems, and new methods of teaching math/calculus. 15 years in industry control systems and power generation industry prior to academic career.

\section{Dr. Aly Farag, University of Louisville}

Aly Farag, Fellow, IEEE and IAPR: received B.S. in EE from Cairo Univ. M.S. in Bioengineering from the Ohio State and the Univ. of Michigan, and PhD in EE from Purdue. He is a Prof. of ECE at the Univ. of Louisville, and director of the Computer Vision \& Image Processing Laboratory, focusing on research and teaching in computer vision, biometrics and biomedical imaging. He introduced over 13 new courses into the ECE curriculum, authored over 400 papers, edited two volumes on deformable models and a textbook on Biomedical Image Analysis (Cambridge Univ. Press, 2014). He graduated over 70 MS and $\mathrm{PhD}$ students, and mentored over 20 postdoctoral researchers. He holds seven US patents on object modeling, computer-aided diagnosis, and visualization. He was lead editor of IEEE-TIFS special issue on Face Recognition in the Wild (December 2014), and co-general chair of ICIP-2009. He is recipient of the University top Awards: Research (1999), Teaching (2009, 2011) and Trustees (2015).

\section{Dr. Asem Ali, University of Louisville}

Asem M. Ali received the M.S. degree in electrical engineering from Assiut University, Asyut, Egypt, in 2002, and the Ph.D. degree in computer engineering from the University of Louisville, Louisville, KY, USA, in 2008, where he was a Post-Doctoral Researcher with the Computer Vision and Image Processing Laboratory from 2008 to 2011. He was an Assistant Professor with the Department of Electrical Engineering, Assiut University from 2011 to 2015. He is currently a Research Scientist with the Computer Vision and Image Processing Laboratory. His research interests include image analysis, machine learning, face recognition, and facial expressions and emotions recognition. He has authored over 40 papers in journals and conferences.

\section{Islam Alkabbany, University of Louisville}

\section{Dr. Marci S. DeCaro, University of Louisville}

Marci DeCaro is an Associate Professor in the Department of Psychological and Brain Sciences at the University of Louisville. Dr. DeCaro earned a PhD in cognitive science from Miami University in 2009 and a US Department of Education-funded postdoctoral fellowship at Vanderbilt University from 2009-2011. She directs the Learning and Performance Lab at the University of Louisville. Her research examines the cognitive mechanisms underlying learning and problem solving in both laboratory and educational contexts, including STEM classrooms.

\section{Dr. Thomas Tretter,}

Thomas Tretter is professor of science education and director of the Gheens Science Hall \& Rauch Planetarium at the University of Louisville. His scholarship includes collaborative efforts with science and engineering faculty targeting retention of STEM majors in entry-level STEM courses. 


\title{
Towards a Multi-dimensional Biometric Approach to Real-time Measurement of Student Engagement in the STEM Classroom
}

\author{
James Christopher Foreman ${ }^{1}$ \\ ${ }^{1}$ Department of Engineering Fundamentals \\ Aly A. Farag ${ }^{2}$, Asem Ali ${ }^{2}$, Islam Alkabbany ${ }^{2}$ \\ ${ }^{2}$ Department of Electrical and Computer Engineering \\ Marci S. DeCaro ${ }^{3}$ \\ ${ }^{3}$ Department of Psychological and Brain Sciences \\ Thomas R. Tretter ${ }^{4}$ \\ ${ }^{4}$ Department of Elementary, Middle \& Secondary Teacher Education \\ University of Louisville, Louisville, KY 40292
}

April 29, 2020

\section{Abstract}

This paper is a work in progress, supported by NSF funds, applied to first-year engineering mathematics courses. An approach to performing a quantitative, objective, and real-time measurement of student engagement in the STEM classroom is proposed to initiate a discussion of the concept and use expert opinions of other faculty to guide progress of the work. This will be realized by an engagement measurement system (EMS). The approach observes biometric data from the students and is multi-dimensional in that it incorporates facial expressions, eye gaze, and hand/head/body movement captured by camera, in addition to pulse captured by a wristband device. From these data, a machine-learning model is trained to classify student engagement. Engagement is classified from behavioral, emotional, and cognitive aspects. The ability to measure student engagement can be used by the instructor to tailor the presentation of material in class, identify course material that engages and disengages with students, and identify students that are engaged or disengaged and at risk of failure. Further, this approach allows quantitative comparison of teaching methods, such as lecture, flipped classrooms, classroom response systems, etc. such that an objective metric can be used to close the loop on teaching evaluation.

\section{Introduction}

Student engagement in the classroom is a paramount concern in the successful learning outcomes of students.[1] Whether a traditional lecture course with passive and didactic 
delivery, or a constructivist course where students construct their learning with active methods, engagement is a key requirement for the student to learn.[2]

Questions that often arise include:

- Are the students engaged? Is engagement increasing or decreasing?

- Which students are dis/engaged?

- Is the course material dis/engaging students?

- Is the teaching method dis/engaging?

The first problem is the inability to measure a student's engagement with the course material. Engagement cannot be improved if it cannot be measured and associated with course concepts, teaching methods, etc. The second problem is subjective evaluation of teaching methods, i.e., feedback based on opinion and observation as opposed to feedback quantitatively determined by analysis. This is demonstrated in Fig. \#1.

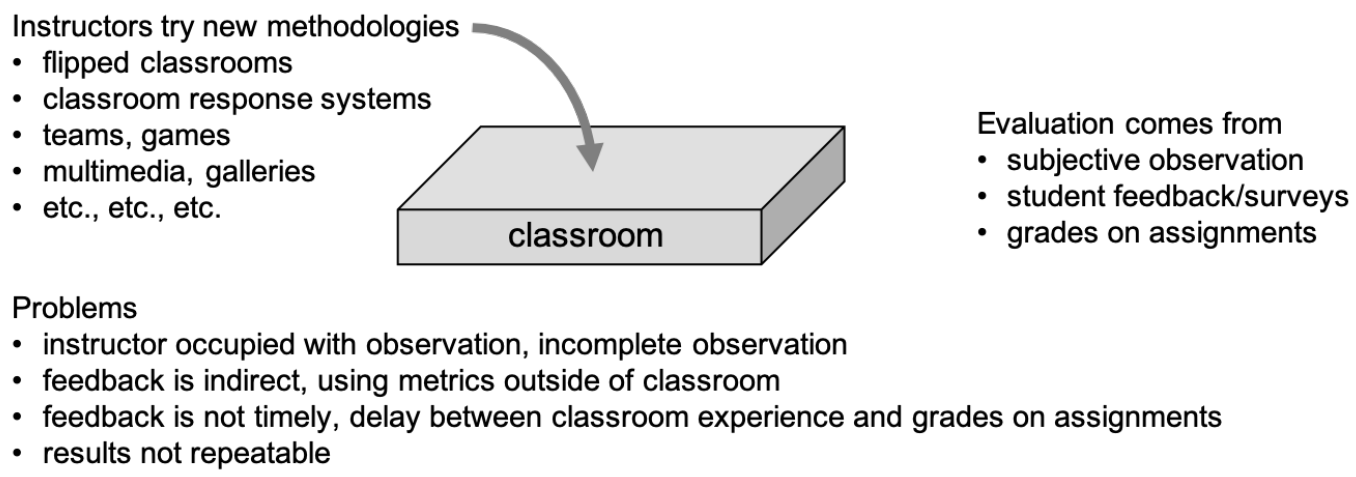

Figure 1: Subjective evaluation of teaching methods.

What is needed is an objective measure of student engagement in the classroom that can be collected in real time, i.e., in situ. This will allow disengaged students to be identified and assisted, letting both instructor and student know immediately when there is an engagement issue. This will also allow a direct comparison between different teaching methods and control groups such that the best methods, and characteristics of methods, can be selected for a given course and student population.

\subsection{Student Engagement in the Classroom}

Student engagement occurs when "students make a psychological investment in learning." [3] This goes beyond motivation [4, p. 85], in which students simply want to learn or have reason to learn, to include the student activities of mental effort and attentiveness for bringing about that learning.

In this paper, engagement is investigated in three components: behavioral, emotional, and cognitive. [5] Behavioral engagement consists of the actions that students take to gain access to the curriculum. These actions include self-directive behaviors outside of class, such as doing homework and studying, as well as other actions, such as shifting in the seat, hand, body, or other sub/conscious movements while observing lectures, and also participating cooperatively in class activities. [6][7] Emotional engagement is broadly defined as how students feel about their learning [8], learning environment [9] and instructors and classmates 
[10]. Emotions include happiness or excitement about learning, boredom or disinterest in the material, or frustration and struggling to understand. Cognitive engagement is the psychological investment in academic achievement such as when students conceptualize, organize, and contemplate in the act of deep learning to understand the course material, e.g.,[11]. These three engagements combine into a student engagement model, further discussed in Section 3.

Teaching is somewhat of a misnomer, in that an instructor cannot place knowledge into a student's mind. It is up to students to learn with instructors having the role to facilitate and guide this learning.[12] For a student to learn, the student must take an active role and responsibility, i.e., psychological investment, in learning.[13] When students are not engaged, e.g., passively listening to instruction, they do not integrate the new information into their long-term memory - learning is shallow and disorganized.[14]

Students may also engage outside of the classroom, such as with homework and study groups, and this should contribute to learning. This is traditionally measured by performance on homework and exams. The focus of this research is engagement in the classroom, which can be observed more directly and in real time, allowing the instructor to tailor delivery of the course content as well as evaluate different teaching methods. This is important, as early detection of students that are at risk or otherwise disengaged can help save these students from failure in the course and withdrawal from school. Students that are not at risk of failure may also become disengaged in the subject or associated major should classroom delivery not adequately capture their engagement. Finally, the high rate of attrition, as much as 50\%, for students in STEM fields [15] is detrimental to students pursuing costly STEM degrees, as well as to society in the reduction of qualified STEM professionals.[16]

\subsection{How Measuring Engagement Facilitates Better Learning}

If engagement is a key metric for student learning and success, then the first step in improving engagement is to be able to measure it. As discussed in Section 1.1, learning depends on the student to taking an active and responsible role in their own learning. If engagement can be measured in situ, e.g., in the classroom, both the instructor and student can objectively observe this metric. Instructors can use the metric to tailor delivery of the course material, spending more or less time on concepts, and move away from ineffective teaching methods and towards effective methods. Students, given this knowledge of their own engagement, can reflect on why they may be disengaged, potentially become motivated to improve their engagement, and communicate effectively with the instructor to seek assistance. Once the

problem of disengagement is identified and associated with specific classroom activities and concepts, both the student and instructor can work together towards a successful learning outcome.

\subsection{How Measuring Engagement Facilitates Better Evaluation of Teaching Methods}

In the past several years, much work has been done to change the dynamics of the classroom environment, such as with revised seating arrangements and multimedia; and of the delivery of course material, such as with flipped classrooms, team-based and peer-assisted learning, 
etc., in an effort to increase academic success, presumably by increasing engagement. In all of these scenarios, evaluation as to the success of the new method largely rests on the evaluation of instructor observation, student surveys, and academic performance. Instructor observation can be subjective, especially when self-evaluating. Further, the instructor is occupied with delivering the course material or facilitating the class, thus not available to focus on observing all of the students nor recording these observations. Student surveys can be biased as students tend to reward entertaining instruction and the conferral of high scores. Students are also not the best assessors of their learning, e.g., students tend to rate their learning lower in constructivist classrooms even when their academic performance is higher, and vice versa for didactic classrooms.[17] Finally, academic performance is not a direct or timely measure of engagement and can be ambiguous.[18] Some students are engaged but struggling, while other students are disengaged, both of whom perform poorly academically. Once assignment scores are determined, it is often too late to engage the student in that material and the student begins to fall behind, resulting in another barrier to engagement.

A quantitative measure closes the loop on the comparison and evaluation of teaching methods, such that classroom sessions can now be engineered to improved engagement. Cognitive engagement is difficult to measure directly [18], and so it is inferred from behavioral and emotional engagements. This is demonstrated in Fig. \#2.

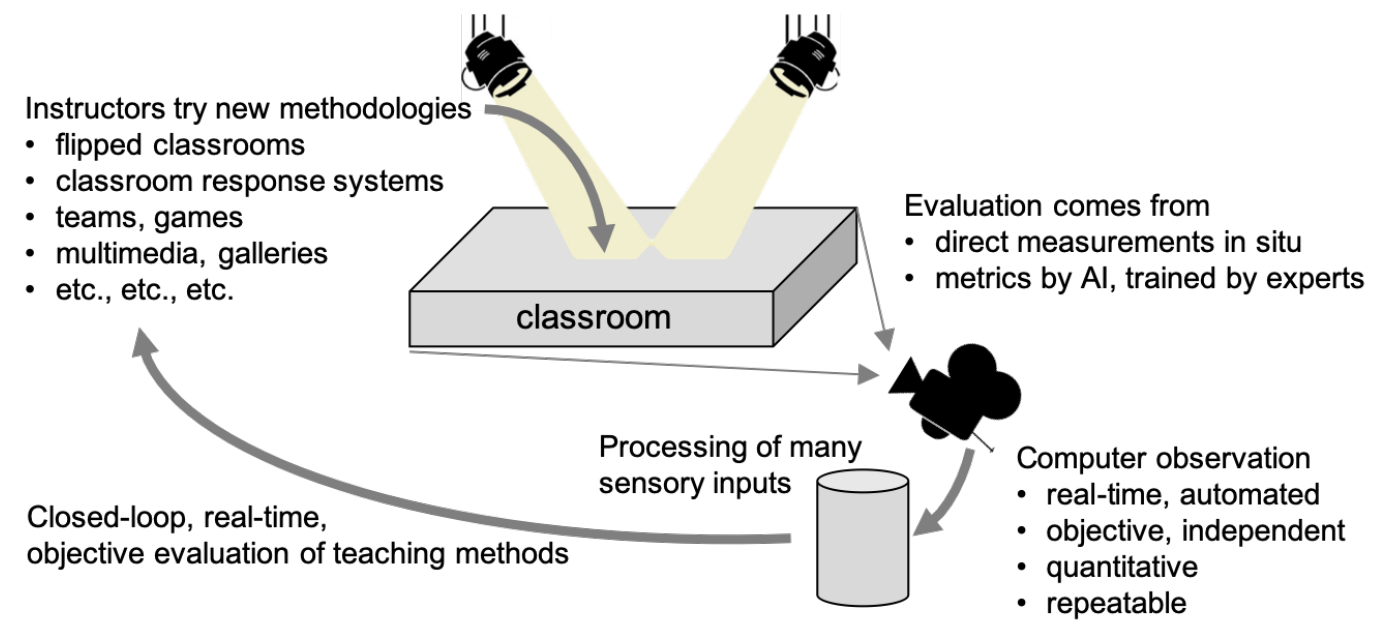

Figure 2: Closing the loop on teaching method evaluation.

\section{Other Efforts in Measuring Student Engagement}

Application of an in situ measurement of student engagement applied to the health professions is given in [19] as STROBE. The application is a methodology of using multiple human expert observers to classify student engagement during classroom sessions. The study notes significant correlation among expert human classroom observers, thus validating conclusions by consensus. As STROBE relies exclusively on human observation, it is not automated and is labor intensive. A similar application to large university classrooms [20] also uses multiple human observers to observe students at regular intervals within a classroom session, defining a protocol to objectify the observer results. Also, [21] applies 
a human observer methodology to courses that are technology mediated, or significantly delivered by technology versus traditional lecture. The Engagement Measurement System (EMS) proposed in this paper seeks to automate such observation using camera capture, computer classification, and validation by experts to train an artificial intelligence (AI) model, replacing human observers to allow real-time measurement of many to all students simultaneously.

An automated system was developed by [22] utilizing eye-gaze tracking collected on each student from which attention and engagement are inferred, which relied on expert human observers for validation. The EMS proposed in this paper collects and integrates many more pieces of information to infer emotional, behavioral, and cognitive engagements and reports these in both a summarized instructor dashboard and more detailed analysis archived for offline use.

\subsection{Previous Work on Classifying Emotion from Facial Expres- sion}

Previous work supports the ability to select facial features from an image of the student's face [23] and classify these [24] by the Facial Action Coding System (FACS) [25] even under pose variations. Using FACS, the expressed emotion of the student can be classified [26], enhanced by the additional data of student behaviors and academic performance integrated into the proposed EMS.[24] Different facial expressions with features selected by image processing and classification by emotion are demonstrated in Fig. \#3.
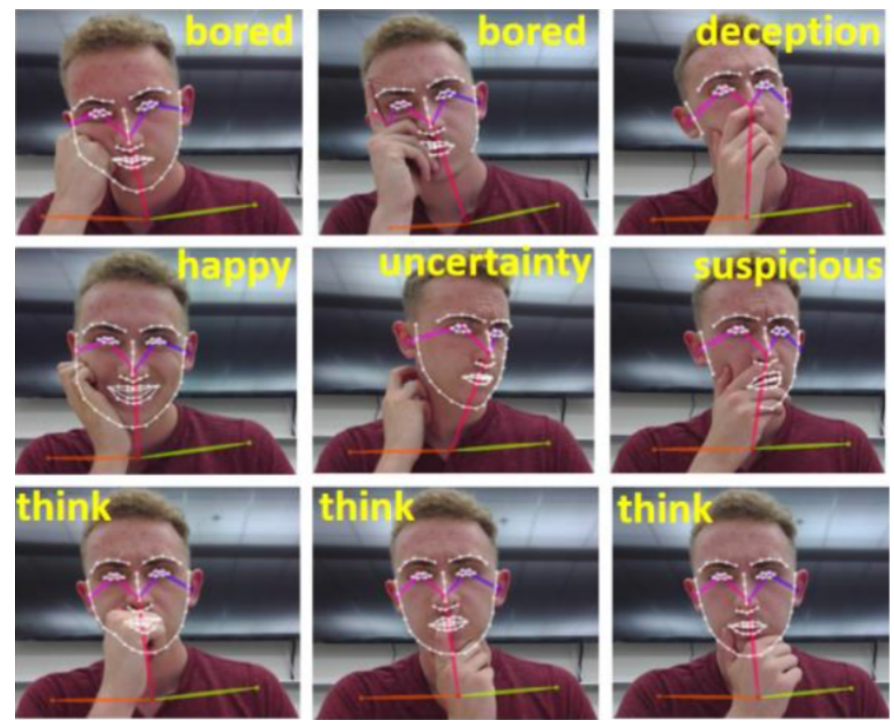

Figure 3: Examples of classification of emotion by expression.

\section{Overall Design of an Engagement Measurement Sys- tem in the Classroom}

The cognitive component of engagement is supported by the behavioral and emotional components as previously discussed 1.1. Student behaviors, such as the actions the stu- 
dent takes to access the curriculum, and the student's emotional state, such as how the student feels about the curriculum, contribute to how well the student learns. Therefore, behavioral and emotional engagement will be directly measured such that these may be used as predictors of cognitive engagement. While the mathematical relationship of how behavioral and emotional engagement maps to cognitive engagement is currently unknown, continuation of this work hopes to uncover those relations as more data are collected. The path of research will proceed as demonstrated in Fig. \#4 as a plan for EMS development.

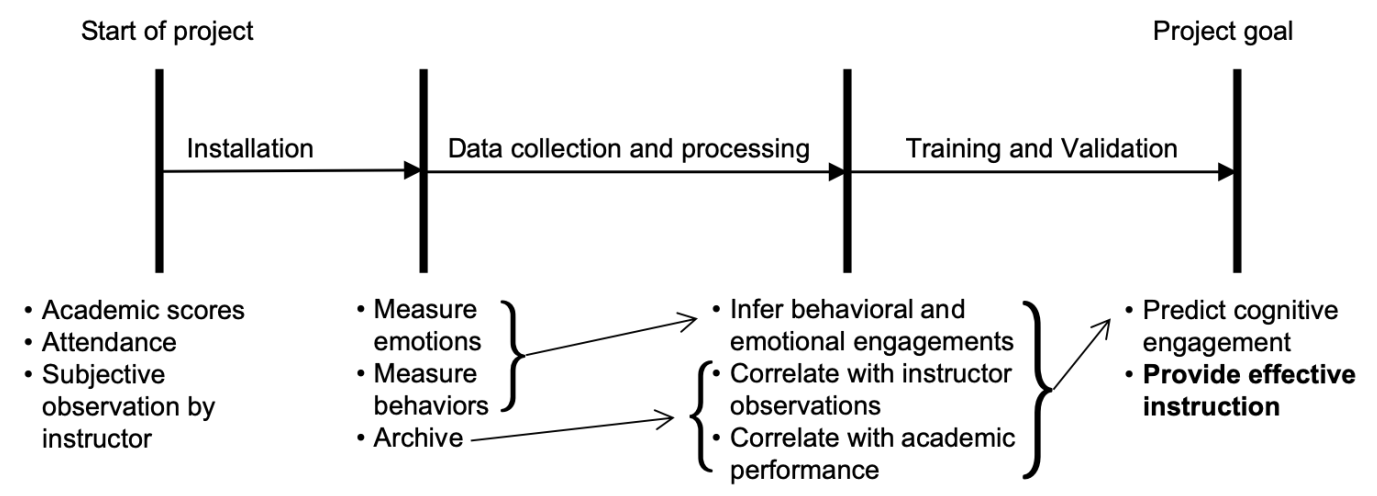

Figure 4: Plan for EMS development.

In Fig. \#4, academic performance (grades), attendance, and occasional subjective observation by the instructor are the resources currently in use for inferring student engagement. By deploying the sensory portion of the EMS, facial expressions, body language and movements, and other biometric data are also collected in real time. The EMS is then trained to determine behavioral and emotional engagement and to further infer cognitive engagement from these. The goal is to use cognitive engagement as a direct metric for student learning and indicate effective instruction.

Part of the data collected will be from assignments, such as homework and exam scores, to represent academic performance, i.e., assignment data. Academic performance is the summative assessment of learning, and can be used as an indicator of cognitive engagement. A student who consistently attends class and completes their homework assignments on time is exhibiting behavioral engagement by choosing this behavior in the course, and this behavior supports cognitive engagement. While the collected assignment data is not used in the live measurement of engagement, it is used in training and validation of the EMS, discussed further in Section 3.2.

Other behaviors include eye gaze/blinking, hand/body motions, postures, etc. Facial expressions can be predictors of emotional engagement, discussed in Section 2.1. By measuring these biometric data, a model can be built to infer cognitive engagement and use this as a predictor of effective instruction, given that cognitive engagement is a requirement of learning as discussed in Section 1.1.

\subsection{Hardware Layout and Software Components}

The EMS hardware layout is demonstrated in Fig. \#5. Each student will make use of either their own laptop camera or a small desk camera dedicated to them. Wristband biometric 
data including heart rate will also be collected and integrated into the classification if determined to be an effective data point. Overview cameras, typically one to a few per classroom, will capture overall student movement and gestures. These biometric data are summarized and sent to a data collection server for analysis. Finally, this analysis is archived in detail, as well as being sent to the instructor in a highly summarized state. This is illustrated in Fig. \#7.

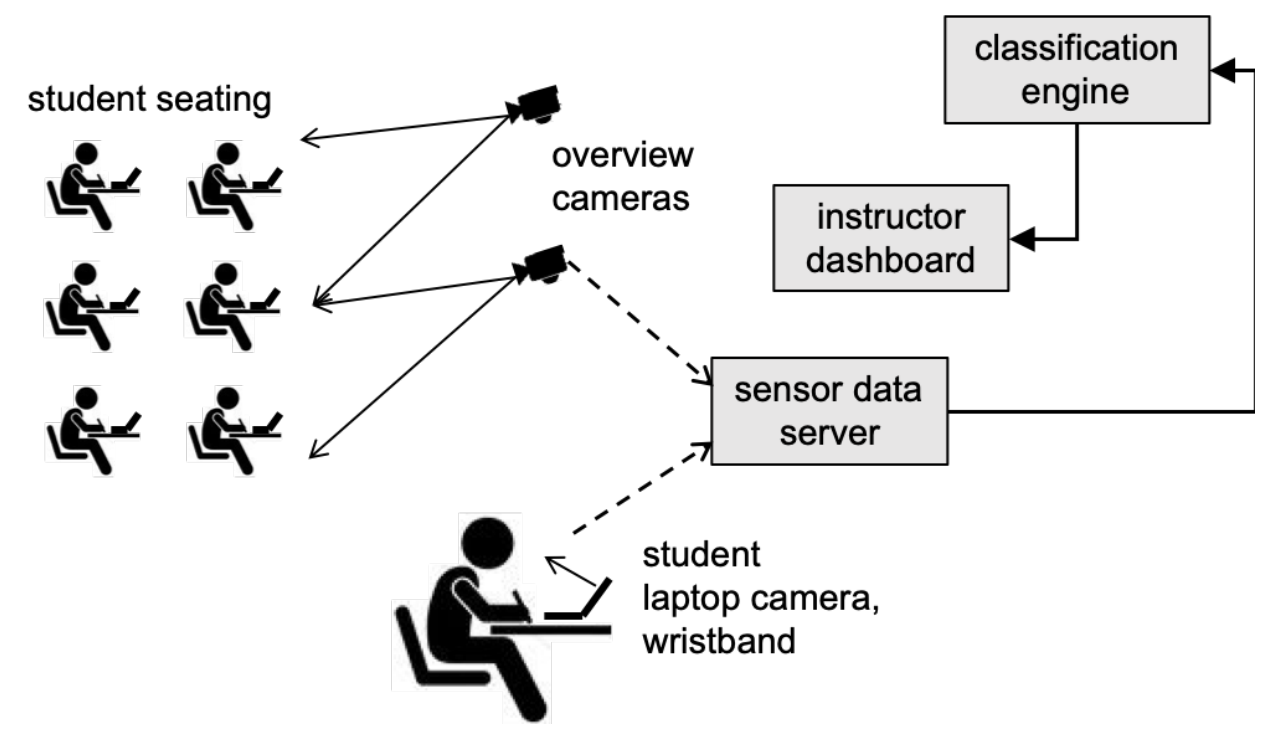

Figure 5: Layout of engagement measurement system in the classroom.

Individual student cameras and wristband data will be collected by a wireless network in the classroom. By using a software client on the student's laptop, the student maintains control over their personally identifying biometric data, such as actual camera images and heart rate, such that only summarized biometric data are sent to the server. This also reduces network load.

The classification engine performs pattern recognition by feature extraction using convolutional layers. These features are then sent to an artificial neural network for classification of emotional and behavioral engagement. Cognitive engagement is further classified by a following artificial neural network layer integrating assignment and attendance data. It may also be the case that some expert observation data as well as student survey data of their perceptions of engagement are also used to guide learning in these artificial neural networks. This is demonstrated in Fig. \#6. 


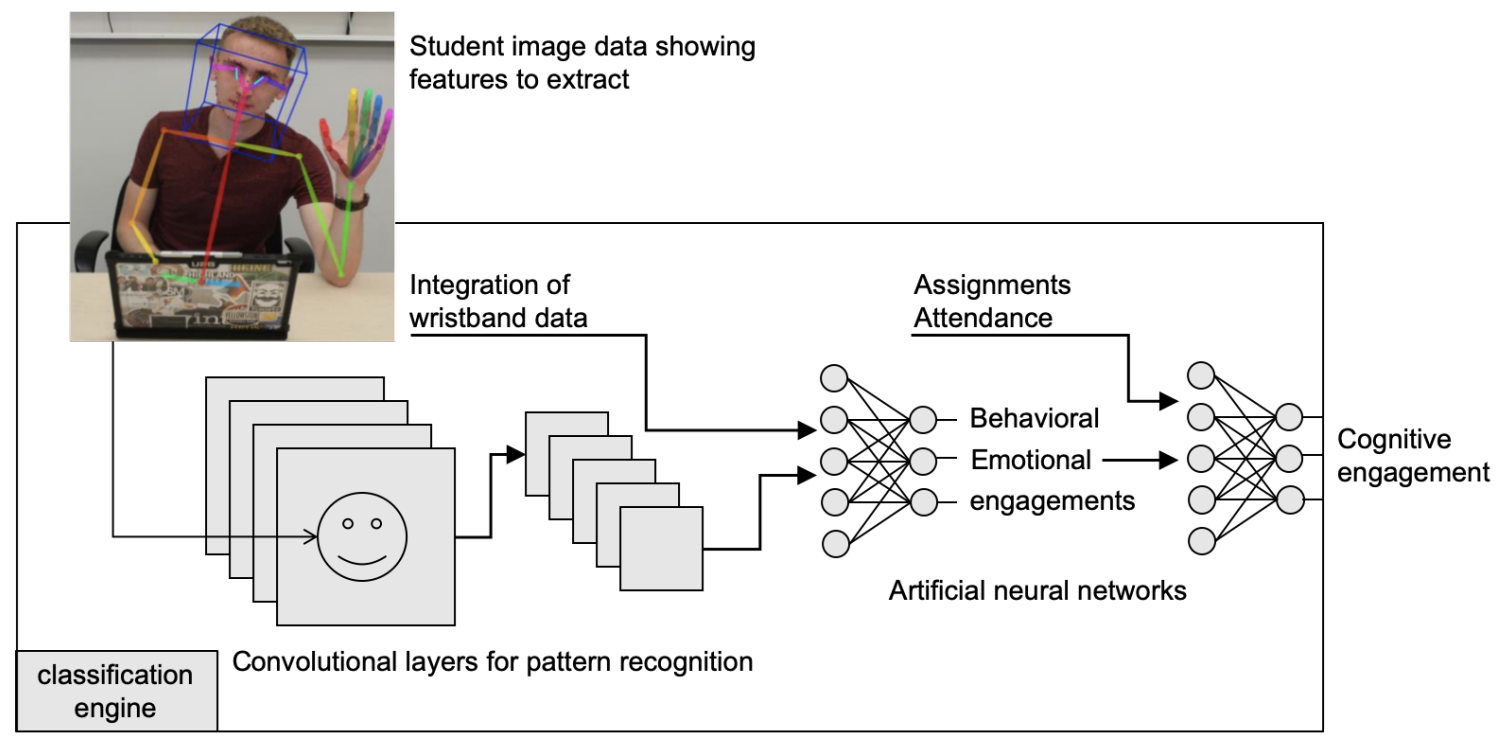

Figure 6: Classification engine using collected data.

As this paper represents a work in progress and the design of the EMS is data driven, the final configuration of the classification engine is reserved for a future paper after live data has influenced the design.

\subsection{Validation}

Validation of the EMS consists of the system's ability to perform the following functions.

1. Accurately capture facial expressions and classify by emotion

2. Accurately capture and classify behaviors

3. Incorporate disparate biometric data (e.g., eye gaze/movement, head/hand/body movements)

4. Incorporate academic performance as assignment data

5. Infer cognitive engagement from these biometric and assignment data

6. Provide effective advisory data to the instructor in situ based on the three types of engagement

7. Archive the data for future analysis

Previous results as described in Section 2.1 with expert analysis can validate items \#1 and \#2. Items \#3 and \#4 are also a function of AI expertise in the incorporation of disparate data types into a classification engine.

Item \#5 is the primary goal and innovation of the EMS. To infer cognitive engagement, multiple human experts in educational instruction, student emotional and behavioral psychology, and course subject matter will all validate the results of the EMS classification from their point of view. These diverse points of view are likely the best approach to validate the EMS as the EMS will itself become an expert observer of the students and classroom. Further, the performance on academic assignments will also be used as a direct correlation with cognitive engagement as these scores (grades) are the current assessment standard. Student surveys will also be planned to get students' conscious awareness of their 
own engagement during the EMS validation. These will provide feedback for the learning algorithms in the EMS.

Item \#6 will be validated by a survey of several instructors in undergraduate STEM courses to determine both the effectiveness and practicality of viewing and utilizing summarized EMS results in the classroom. The ability to successfully use the instructor dashboard will also be validated by the improvement in engagements scores, i.e., self validating by feedback. Item \#7 will be a straightforward manner of proving that the captured data can be archived.

\subsection{How to Use the EMS}

The EMS is to be used in situ as an advisor to the instructor during classroom sessions. A very quick glance can guide the instructor on one of a few paths during material delivery. Additionally, the EMS can be utilized in much more detail after class, i.e., offline, with the ability to examine responses on the individual student level and analyzing with respect to assignment scores.

\subsubsection{Inside the Classroom with the Instructor Dashboard}

The instructor dashboard will be an instrument to display student engagement data as well as EMS functionality in real time during classroom sessions. Because the instructor will be primarily occupied with managing the classroom and facilitating the delivery of course material, the instructor dashboard needs to be very simple and direct in communicating student engagement. Ideally, the instructor should be able to obtain meaningful data at a glance that is sufficient to guide delivery. For example, a quick glance that indicates cognitive engagement is falling could signal the instructor to either move on to the next topic as students are getting bored or that the students are not understanding the current material. Secondary information on behavioral and emotional states could help determine which of these is most prevalent and the best action to take. Other indications might be increasing engagement, which would signal positive feedback to the instructor regarding the current method of delivery in the classroom. Fig. \#7.

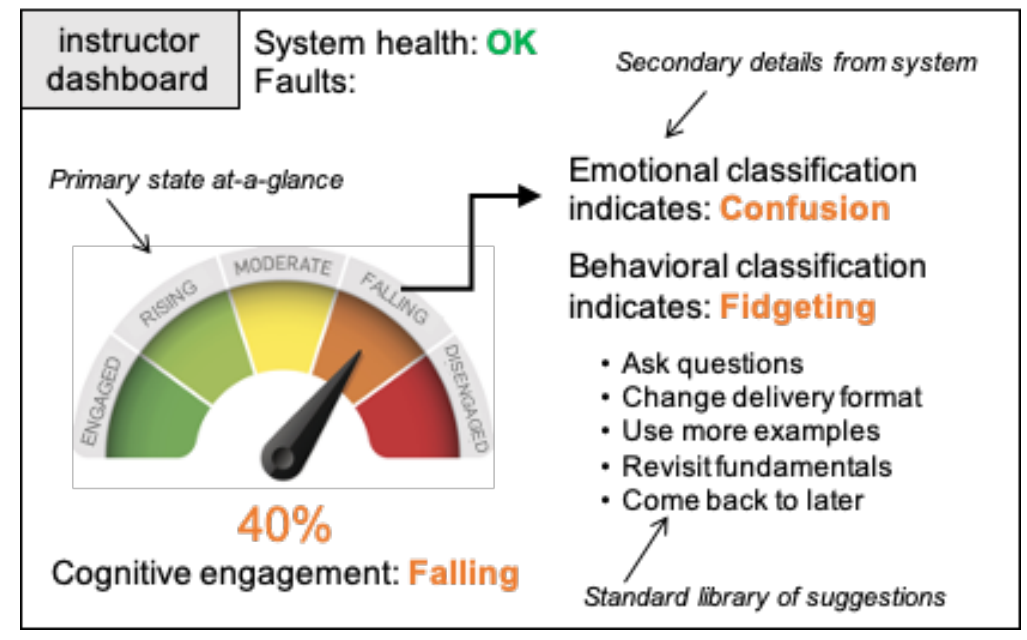

Figure 7: Example of instructor dashboard. 


\subsubsection{Outside of the Classroom with Offline Review}

The collected biometric data will be archived during the semester. This will allow the instructor to go back and replay the classroom sessions, correlate material delivered with academic performance on corresponding assignments, and analyze with the benefit of live engagement scores. Assignment data will also be retained for training and validation of the EMS predictions to facilitate future design iterations of the course and further observation by educational researchers, e.g., to examine what forms of instruction or active learning best engage students.

In later iterations of the EMS, the students will also be given their personal live engagement data versus time during the classroom session. This will allow students to reflect on their personal engagement and give them insight into where they are becoming disengaged.

\section{Final Discussion}

The goal of this paper is to initiate discussion of the approach proposed in the abstract to develop a system to measure engagement in the classroom. Feedback of peer instructors in the STEM fields will be solicited to guide initial exploration. Initial deployments of the system will provide live classroom data from which to observe conclusions in the future.

The fundamental goal of using the engagement measurement system (EMS) is to improve student learning. Understanding engagement versus different classroom scenarios will help in selecting successful classroom scenarios, e.g., flipped classroom, multimedia, interactive learning exercises, etc. Academic performance on assignments and student feedback will serve to validate these new measurements against existing metrics in use. Once developed, a hardware solution can be feasibly deployed by other learning institutions in terms of readily available camera and computer hardware, coupled with software classification developed by this work. It is expected that application would extend to vocational/workforce training as well as subjects outside of STEM.

The previous success in emotional and behavioral classifications serve as a foundation on which to explore the relation of these types of engagement in supporting cognitive engagement. At the very least, both students and instructor will have much more detailed information about how the course proceeded and how the classroom sessions were perceived and presented. The goal will be to ensure that this detailed information improves cognitive engagement and that this supports student success in achieving learning outcomes.

\subsection{Supporting Funding}

Current funding is provided by an exploratory grant from NSF Improving Undergraduate STEM Education (IUSE). The goal of this three-year exploration is to determine if such a system as the EMS can be built to collect this data in real time within the classroom and accurately capture behavioral and emotional engagements. Also to be explored is the integration of disparate data types such as image data, heart rate, academic performance, etc., within an AI classification system. Final efforts on the project will include preliminary evaluation of the ability to infer cognitive engagement from behavioral and emotional engagements. As demonstrated in previous work, classifying emotions and behaviors is pos- 
sible to some extent, and psychological work has established the link between emotional and behavioral engagement with cognitive engagement.[27][28]

\section{References}

[1] Matthew J Bundick, Russell J Quaglia, Michael J Corso, and Dawn E Haywood. Promoting student engagement in the classroom. Teachers College Record, 116(4):n4, 2014.

[2] Selim Gunuc and Abdullah Kuzu. Student engagement scale: development, reliability and validity. Assessment 65 Evaluation in Higher Education, 40(4):587-610, 2015.

[3] Fred M Newmann. Classroom thoughtfulness. Student engagement and achievement in American secondary schools, pages 62-91, 1992.

[4] Shlomo Sharan, Hanna Shachar, and Tamar Levine. The innovative school: Organization and instruction. Greenwood Publishing Group, 1999.

[5] Jennifer A Fredricks, Phyllis C Blumenfeld, and Alison H Paris. School engagement: Potential of the concept, state of the evidence. Review of educational research, 74(1):59-109, 2004.

[6] Ning Hao, Hua Xue, Huan Yuan, Qing Wang, and Mark A Runco. Enhancing creativity: Proper body posture meets proper emotion. Acta psychologica, 173:32-40, 2017.

[7] Valentina Rita Andolfi, Chiara Di Nuzzo, and Alessandro Antonietti. Opening the mind through the body: The effects of posture on creative processes. Thinking Skills and Creativity, 24:20-28, 2017.

[8] Ellen A Skinner and Michael J Belmont. Motivation in the classroom: Reciprocal effects of teacher behavior and student engagement across the school year. Journal of educational psychology, 85(4):571, 1993.

[9] Kristin E Voelkl. Identification with school. American Journal of Education, 105(3):294-318, 1997.

[10] James J Appleton, Sandra L Christenson, Dongjin Kim, and Amy L Reschly. Measuring cognitive and psychological engagement: Validation of the student engagement instrument. Journal of school psychology, 44(5):427-445, 2006.

[11] Michelene TH Chi and Ruth Wylie. The icap framework: Linking cognitive engagement to active learning outcomes. Educational psychologist, 49(4):219-243, 2014.

[12] G DeBoer. A history of ideas in science education: Implications for practice. 1234 amsterdam avenue. New York, NY, 10027, 1991. 
[13] S Lamborn, F Newmann, and G Wehlage. The significance and sources of student engagement. Student engagement and achievement in American secondary schools, pages 11-39, 1992.

[14] Michelene T. H. Chi and Ruth Wylie. The icap framework: Linking cognitive engagement to active learning outcomes. Educational Psychologist, 49(4):219-243, 2014.

[15] Yujing Chen, Aditya Johri, and Huzefa Rangwala. Running out of stem: a comparative study across stem majors of college students at-risk of dropping out early. In Proceedings of the 8th International Conference on Learning Analytics and Knowledge, pages 270-279. ACM, 2018.

[16] Xianglei Chen. Stem attrition: College students' paths into and out of stem fields. statistical analysis report. nces 2014-001. National Center for Education Statistics, 2013.

[17] Louis Deslauriers, Logan S McCarty, Kelly Miller, Kristina Callaghan, and Greg Kestin. Measuring actual learning versus feeling of learning in response to being actively engaged in the classroom. Proceedings of the National Academy of Sciences, 116(39):19251-19257, 2019.

[18] Heather L O'Brien and Karon E MacLean. Measuring the user engagement process. In Digital Life New World Conference, Boston, MA, 2009.

[19] Kimberly J O’Malley, Betty Jeanne Moran, Paul Haidet, Charles L Seidel, Virginia Schneider, Robert O Morgan, P Adam Kelly, and Boyd Richards. Validation of an observation instrument for measuring student engagement in health professions settings. Evaluation 80 the health professions, 26(1):86-103, 2003.

[20] Erin S Lane and Sara E Harris. A new tool for measuring student behavioral engagement in large university classes. Journal of College Science Teaching, 44(6):83-91, 2015 .

[21] Curtis R Henrie, Lisa R Halverson, and Charles R Graham. Measuring student engagement in technology-mediated learning: A review. Computers \& Education, 90:36-53, 2015.

[22] Jonathan Bidwell and Henry Fuchs. Classroom analytics: Measuring student engagement with automated gaze tracking. Behav Res Methods, 49:113, 2011.

[23] Eslam Mostafa, Asem A Ali, Ahmed Shalaby, and Aly Farag. A facial features detector integrating holistic facial information and part-based model. In Proceedings of the IEEE Conference on Computer Vision and Pattern Recognition Workshops, pages 9399, 2015.

[24] Asem M Ali, Islam Alkabbany, Amal Farag, Ian Bennett, and Aly Farag. Facial action units detection under pose variations using deep regions learning. In 2017 Seventh International Conference on Affective Computing and Intelligent Interaction (ACII), pages 395-400. IEEE, 2017. 
[25] E Friesen and Paul Ekman. Facial action coding system: a technique for the measurement of facial movement. Palo Alto, 3, 1978.

[26] Islam Alkabbany, Asem Ali, Amal Farag, Ian Bennett, Mohamad Ghanoum, and Aly Farag. Measuring student engagement level using facial information. In 2019 IEEE International Conference on Image Processing (ICIP), pages 3337-3341. IEEE, 2019.

[27] Yibing Li and Richard M Lerner. Interrelations of behavioral, emotional, and cognitive school engagement in high school students. Journal of Youth and Adolescence, 42(1):20-32, 2013.

[28] Hao Lei, Yunhuo Cui, and Wenye Zhou. Relationships between student engagement and academic achievement: A meta-analysis. Social Behavior and Personality: an international journal, 46(3):517-528, 2018. 